\title{
Short Radius Centrifuges - A New Approach for Life Science Experiments Under Hyper-g Conditions for Applications in Space and Beyond
}

\author{
Vanja Zander ${ }^{*}$, , Ralf Anken ${ }^{1}$, Thomas Pesquet ${ }^{2}$, Sonja Brungs ${ }^{1}$ and Joachim Latsch ${ }^{3}$ \\ ${ }^{1}$ Institute of Aerospace Medicine, German Aerospace Center DLR, Linder Hoehe, D-51147 Cologne, Germany \\ ${ }^{2}$ European Astronaut Center, European Space Agency, Linder Hoehe, D-51147 Cologne, Germany \\ ${ }^{3}$ Institute of Cardiology and Sportmedicine, German Sport University Cologne, Am Sportpark Muengersdorf 6, D-50933 \\ Cologne, Germany
}

Received: 25 February 2013; Revised: 01 April 2013; Accepted: 10 April 2013

\begin{abstract}
A broad variety of countermeasures on the effects of weightlessness on human physiology have been developed and applied in the course of space exploration. Devices like treadmills, stretch ropes etc. have several disadvantages in common: they require a significant amount of crew time and they may not efficiently counteract the degradation of physiological structures and cellular functions. Some methods even include potentially painful or uncomfortable procedures for the astronauts. Thus, the application of Artificial Gravity (AG) generated by short radius centrifuges (they fit into space vessels) has been discussed and proposed by a number of scientists and space agencies as an alternative countermeasure during long-term space missions. Although there is a profound knowledge concerning, e.g., the cardiovascular system and immune responses acquired on long radius centrifuges, there is a remarkable lack of knowledge concerning the same issues on devices operating with short radius. In strict contrast to long radius centrifuges, there is a significant gravity gradient in the head-to-toe axis which comes along with the short radius and higher relative rotation velocity. Thus it is of utmost importance to continue investigating the effects of AG, especially by use of short radius centrifuges.
\end{abstract}

The Short Arm Human Centrifuge (SAHC) at the German Aerospace Center (DLR) in Cologne, Germany, is the most advanced type of short radius centrifuges presently commercially available. Experience gained so far using the SAHC at DLR revealed that future projects on centrifuge devices with short radius should aim at a clear identification of the threshold level of the $g$-load, which is necessary to efficiently counteract the degradation of physical structures and an efficient support of cellular functions. A satisfying result would be combined countermeasure methods applied at a threshold concerning g-load and exposition time in the course of long-term sojourn in microgravity. Another future control or monitoring method to exactly dose AG training is heart rate variability, which offers an insight into neurovegetative and cardiovascular regulation.

Centrifuges like the SAHC are also useful platforms to accommodate small biological experiments, e.g., experiments addressing the response of cultured cells to hypergravity.

Here, we briefly review the issue of short radius centrifuges and also address our experience hitherto gained during a number of scientific projects carried out at the SAHC at DLR.

Keywords: Centrifuge, hyper-g, cardiovascular system, neurovestibular system, countermeasure, artificial gravity.

\section{INTRODUCTION}

Long radius centrifuges have been amongst the most important training facilities for military jet pilots and astronauts in the course of the last seven decades. Performing tight turn maneuvers with high speed in airplanes and accelerations during take-off and landing in space vessels, respectively, expose the human physiology to high and continuous $g$-loads of up to $9 g$ and for short periods of time even more. In contrast to Long Arm Human Centrifuges, Short Arm Human Centrifuge (SAHC) devices

*Address correspondence to this author at the Institute of Movement and Neurosciences German Sport University Cologne, Am Sportpark Müngersdorf, D-50933 Cologne, Germany; Tel: +49 2214982 5040; Fax: +49 2214982 906; E-mail: v.zander@dshs-koeln.de operate with a considerably shorter radius and therefore higher relative velocity, which is necessary to generate adequate $g$-loads for experiments, tests and training. These devices moved into focus of space agencies, such as the European Space Agency (ESA), after the manning of the International Space Station (ISS) in November 2000 (ISS-E 1) and due to on-going plans for interplanetary missions to the Moon and Mars.

Due to significant health and potentially life threatening physiological alterations such as cardiovascular deconditioning, alteration of blood pressure (BP) regulation and degression of bone density, which astronauts experience in the course of space missions, efficient countermeasures must be applied in a timely manner [1]. Various sorts of countermeasures (e.g. [2,3]) have been tested and applied 
during space exploration in the course of the $20^{\text {th }}$ century and the first decade of the $21^{\text {st }}$ century. Fixed treadmills, pneumatical resistance devices [2], low body negative pressure (LBNP) devices, exercises with stretch ropes, modified bicycles and self-propelled centrifuge devices [3] are in use in order to prevent excessive degradation of physiological structures and recent patents in the field are included.

All countermeasures mentioned above have something crucial in common: they demand a remarkable share of crew time and resulted to be inefficient to counteract general degradation of physiological structures. Thus, the application of AG generated by short radius centrifuges is under intense assessment in Europe and in other parts of the world [4].

The rotation of at least one subject in supine or sitting position can generate a centrifugal force which simulates the gravity-induced physiological effects while standing at a certain $g$-load. Apart from application of short radius centrifuges in space these devices could be installed on interplanetary destinations such as Mars and the Moon, since the gravity on Mars is only 1/3 compared to gravity on Earth. Besides the application of $\mathrm{AG}$ in space related research centrifuges with short radius ([e.g. 3]) could also be beneficial in the field of sports medicine and competitive sports, rehabilitation and therapy of temporary or permanently immobilized persons [5]. However, there is still a remarkable lack of knowledge concerning the effects of AG generated by a short radius centrifuge and especially the effect of the gravitational gradient on haemodynamics and cardio-vegetative regulation in humans [6].

Therefore, one major objective of future research projects involving short radius centrifuges must be to achieve a basic description and essential knowledge of AG effects/the gravitational gradient effect generated by such a device on the cardiovascular system and the autonomous nervous system (ANS). In particular, questions concerning the heart rate (HR), heart rate variability (HRV) and $\mathrm{BP}$ regulation as well as aortic and carotid baroreceptor function should be addressed. The answer to those questions may consequently lead to an optimized training procedure, as far as $g$-load and exposition time are concerned, on a short radius centrifuge for persons designated to participate in space missions.

\section{TECHNICAL ASPECTS}

\section{The Concept of Short Radius Centrifuges}

There are a number of differences between long radius and short radius centrifuge (Figs. 1-3) devices, as far as rotation velocity, achievable $g$-load, gravity gradient, position of the subject etc. are concerned. Short radius centrifuges require a higher rotation velocity in order to generate a $g$-load adequate to perform training and experiments in AG. There is a severe side-effect of the higher rotation velocity though. Alongside the $A G$ a significant gravitation gradient is generated in the course of centrifugation. Since the head of the subject is oriented towards the stator of the centrifuge and the feet are oriented towards the outer perimeter of the nacelle (Fig. 3), there is a gradient in the gravitational field along the head-to-toe axis of the subject on a short radius centrifuge. This gradient requires further assessment, since it has a tremendous impact on the cardiovascular system during application of AG. Additionally the short arm centrifuges generate significant Coriolis forces and motion sickness stimuli if the head is moved, at least until adaptation occurs. Nevertheless, studies on adaptation have shown successful adaptation by most subjects to head movements even at high $g$-load [5].

The major reason why short radius centrifuges are particularly attractive is the diameter of standard space vessels, which is sufficient to contain such a device. A $2 \mathrm{~m}$ radius $A G$ device would permit subjects to stand upright and even to perform exercises within the device. Some short radius centrifuges are designed to be self-propelled by a pedaling subject. The active exercise on an ergometer bicycle while being exposed to AG with a significant gravity gradient in the head-to-toe axis should protect the subjects from syncope.

\section{Technical Specification of the Short Arm Human Centrifuge (SAHC) at the DLR Institute of Aerospace Medicine}

Currently, there is one short radius centrifuge at the German Aerospace Center (DLR) in Cologne active, called Short Arm Human Centrifuge (SAHC - TN-0007-VE, QinetiQ Company, Antwerp (Belgium). This kind of short radius centrifuge is the most advanced type which is presently commercially available. Another sample exists at the Institute for Space Medicine and Physiology (MEDES) in Toulouse (France).

With this centrifuge provided by ESA and operated by DLR, test subjects can be exposed to various accelerations in supine and sitting position up to $4.7 \mathrm{~g}$ at the level of feet. The research activities are focused on the development of countermeasures against gravity-induced physiological changes and the subsequent medical risks. Although the test subjects shall feel comfortable during centrifuge experiments and the scientist's requirements are taken into account, subject's safety is of utmost importance. This is guaranteed by continuous monitoring of vital signs by a physician, the so called Medical Monitor (MM) (Fig. 3) situated in the Centrifuge Control Room next to the Centrifuge Room (Fig. 2). At the same time SAHC's technical performance is controlled by the Centrifuge Operator (CO). The experimental lead is performed by a research member of staff, the so called Experiment Operator (ExO).

On the SAHC, two test subjects in identical position (supine or sitting) can be spun at the same time. In both positions, the inclination of the nacelle is variable between $10^{\circ}$ and $+90^{\circ}$ (seat) or $87^{\circ}$ (bed). Additional equipment for scientific assessment and ergometric exercise can be implemented according to the scientist's requirements.

\section{Scientific Scope}

- Development of effective countermeasures against cardiovascular and neuromuscular degradation in human physiology.

- Investigation of effects of AG on the cardiovascular and the neurovestibular system. 


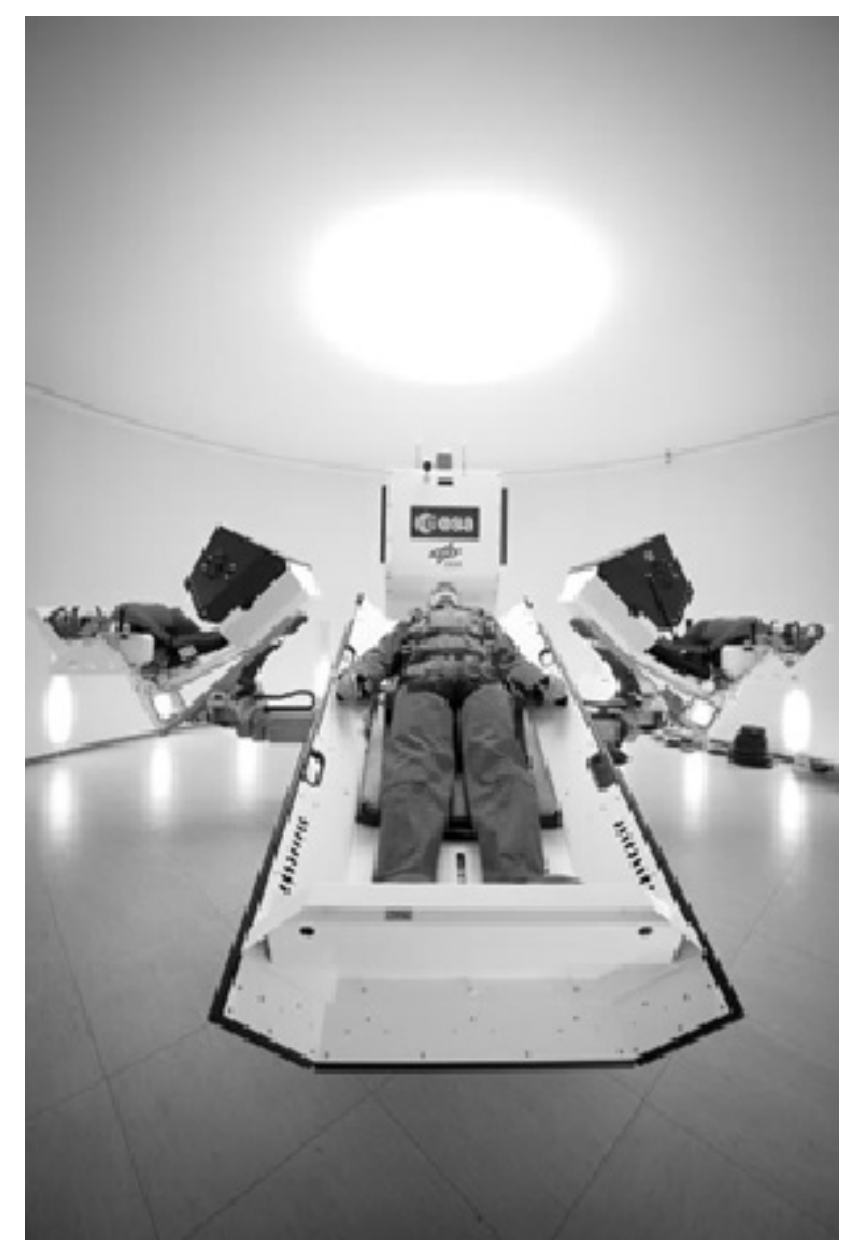

Fig. (1). Short Arm Human Centrifuge (SAHC) at the German Aerospace Center (DLR) in Cologne, Germany.
- Assessment of orthostatic and vestibular tolerance of pilots, parabonauts and astronauts.

- Fundamental research, e.g., with respect to osteoporosis and muscle atrophy.

- $\quad$ SAHC as an instrument for clinical and experimental studies.

- $\quad$ Research in the field of cell physiology (using particular devices to hold experimental setups for, e.g., biotechnological or cell physiological experiments, fitted to the nacelles).

\section{Technical Data}

Beginning in spring 2011, six scientific projects have been performed at the SAHC at DLR. The first study to be performed was the SAHC - Coagulation Study (SAHC COA), which assessed the coagulation parameters in the blood prior and post a constant application of AG ( $2 g$ at the level of feet) on behalf of the DLR. The second study, called SAHC - Cognition Study (SAHC - COG) was performed on behalf the German Sport University Cologne. That project assessed the cognition and the perfusion of the human brain prior, during and post application of AG by measuring the activity of the brain by using non-invasive methods such as Electroencephalography (EEG) and Near-Infrared Spectroscopy (NIRS). Further studies have been performed in cooperation with the Charite - Universtitätsmedizin Berlin, The University of Applied Sciences Bonn, and the University of Bonn. All studies mentioned above were performed using programmed $g$-load profiles and according to the scientists requirements [7].

Regarding operations, these studies led to a variety of important experiences concerning the utilizability of this particular type of centrifuge, which are summarized in the

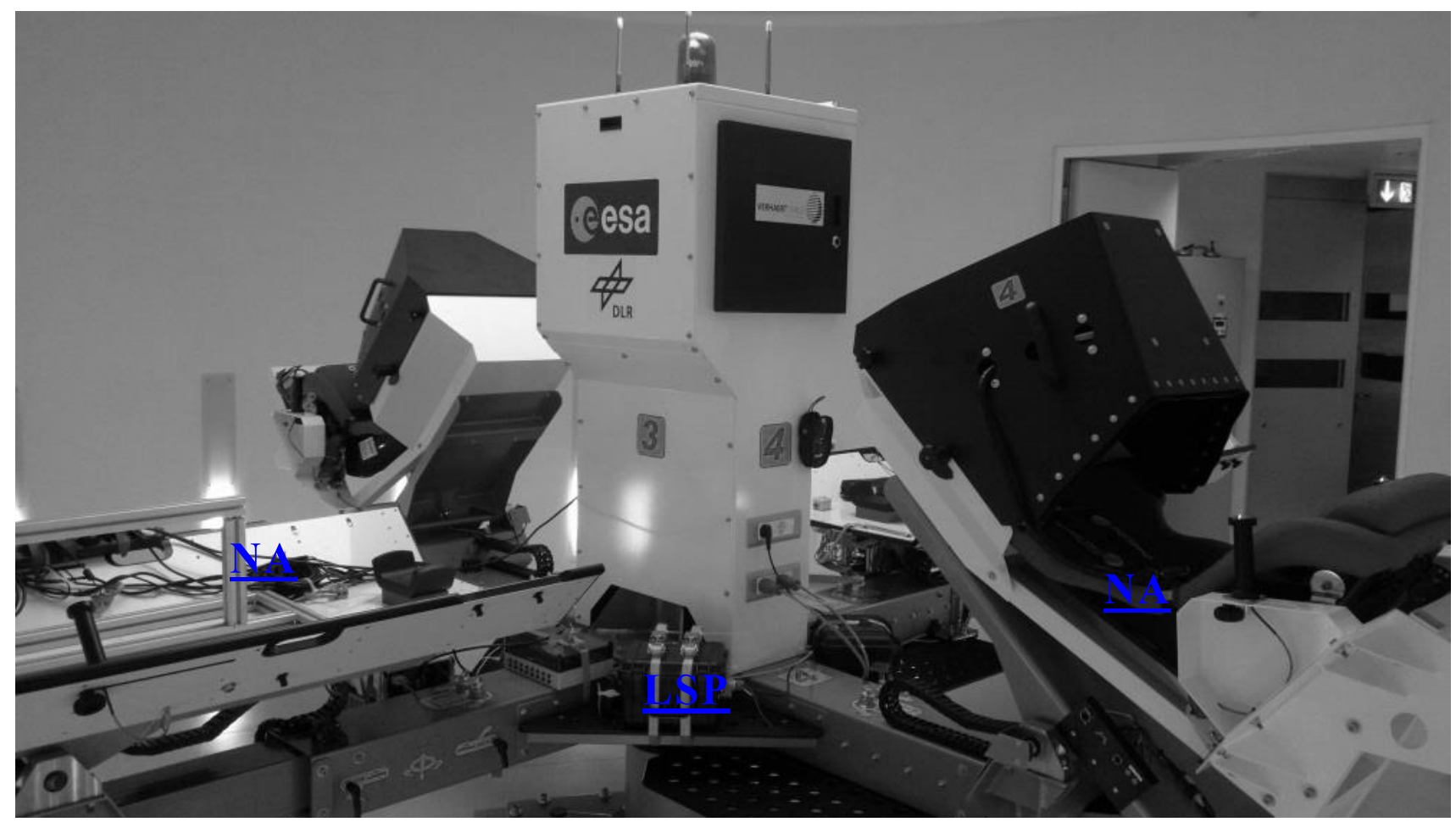

Fig. (2). Short Arm Human Centrifuge (SAHC), Life Science Platforms (LSP) and nacelles (NA) of the SAHC. 


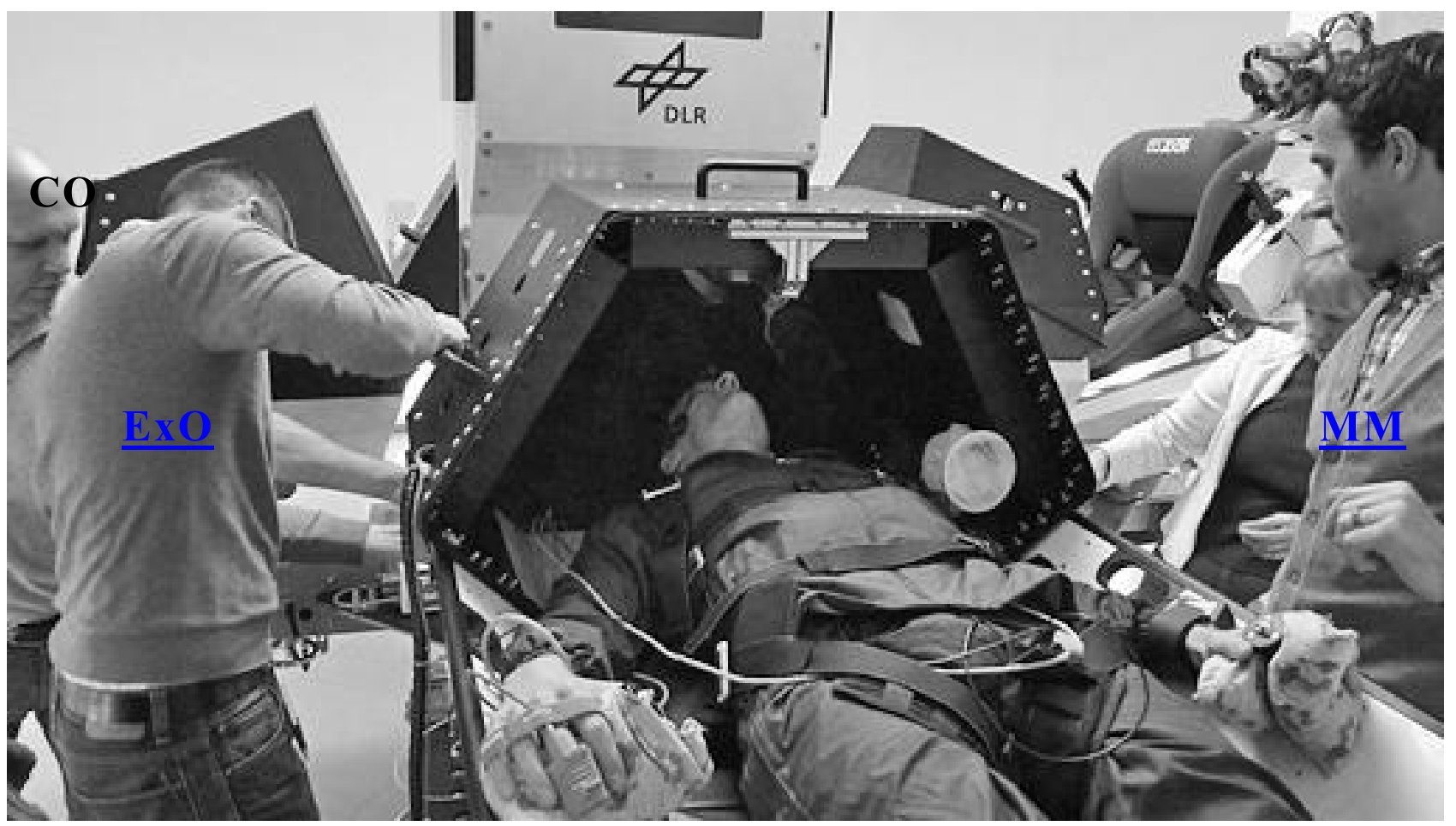

Fig. (3). Short Arm Human Centrifuge (SAHC) Subject in preparation for the run by the Medical Monitor (MM), the Experiment Operator $(\mathrm{ExO})$ and the Centrifuge Operator $(\mathrm{CO})$.

following. Though a budding device, which proved its capability of applying exact $g$-loads by using programmed profiles during complex scientific studies, there are drawbacks in the SAHC design as far as modification of nacelles, payload capacity of the nacelles, payload capacity of the life science platforms (LSP), variety of installable scientific equipment, capability of monitoring two subjects at a time and reachable $g$-load are concerned. Additionally, during the studies mentioned above, subjects had to be spun one at a time, since there is no way to reduce the $g$-load individually for one subject. Concerning the g-load, the lesson has been learnt that $2 g$ over an average exposition time of 30 minutes are well capable of initiating syncope. With the new short radius centrifuge (: envifuge), which is integrated into the new-built : envihab complex at the DLR, the experiences made with the SAHC were used to build a new generation of short radius centrifuges. The : envifuge is capable of generating a $g$-load of up to $6.4 g$ at the outer perimeter. With the new grid structure built above the nacelles and a remote controlled robotic arm, the : envifuge is capable of performing a number of new assessments. At the same time the nacelles can be individually moved away towards the stator in the course of centrifugation. Therefore, the $g$-load can be adjusted automatically if necessary.

\section{Application of AG in Space}

Outer space (defined by an altitude of more than $100 \mathrm{~km}$ ) is one of the harshest environments one may find and certainly not hospitable to the human physiology. Vacuum, extreme temperatures and radiation are out of scope of this

\section{Table 1. SAHC Technical Specifications}

\begin{tabular}{|c|c|}
\hline max. radius (outer perimeter) & $2.82 \mathrm{~m}$ \\
\hline max. centrifugal acceleration & $4.7 g$ at the outer perimeter \\
\hline min, centrifugal acceleration & $0.1 g$ at the outer perimeter \\
\hline max. $g$-acceleration rate & $0.25 \mathrm{~g} / \mathrm{s}$ \\
\hline max. rotor revolution & $40 \mathrm{rpm}$ \\
\hline min. rotor revolution & $5.7 \mathrm{rpm}$ \\
\hline number and type of nacelles & $2 \mathrm{beds}$ \\
& 2 seats \\
\hline max. payload & $550 \mathrm{~kg}$ \\
\hline
\end{tabular}

manuscript, but long-term spaceflight has further challenges in store for the human physiology as well as for the technical equipment aboard a space vessel, i.e., the effect of weightlessness. Weightlessness has a number of severe effects on human physiology, some of which can be mitigated by application of AG generated by a short radius centrifuge or combined countermeasures. However, no formal human $\mathrm{AG}$ experiments or training were performed in space during the first 50 years of space exploration.

During the first spaceflight concerns focused on the "space adaptation syndrome" since the missions in weightlessness lasted only for few days. Degradation of the cardiovascular system and the musculoskeletal system were not assessed before the Skylab mission in the course of the early 1970s [8]. During the Skylab mission the crew used the 
empty space compartment to run around the curved inner hull. One may say the crew generated self-propelled AG by running. Though no relevant AG experiments have been performed, on few occasions centrifugation for other purposes has produced a measure of centripetal acceleration. In the course of the First International Microgravity Laboratory (IML-1) Spacelab mission, subjects were turned on a rotator with their head $0.5 \mathrm{~m}$ off the stator, generating a $g$-load of $-0.22 g$ at the level of head and approximately $+0.36 \mathrm{~g}$ at the level of feet [9]. In contrast to IML-1, during the Neurolab Spacelab mission four subjects developed some sort of immunity to orthostatic instability. At the same time they did not show any common decrease in vestibular sensitivity to tilt $[10,11]$. Further assessments were performed in the course of long-duration mission aboard the space stations Saljut and Mir [12].

\section{Technical Requirements of the ISS Environment to a Short Radius Centrifuge}

The ISS is a complex assembly of cylindrical modules. It weighs approximately $250 \mathrm{t}$ and measures $108.5 \times 72.8 \mathrm{~m}$ for the total habitat volume of $837 \mathrm{~m}^{3}$. The diameters of the modules vary slightly between $4.28 \mathrm{~m}$ and $4.72 \mathrm{~m}$ and their length is comprised, depending on the module, between 6.5 $\mathrm{m}$ and $11.2 \mathrm{~m}$ (not considering the airlocks). The ISS flies in the vacuum of space while maintaining an internal pressure of $103.3 \mathrm{kPa}$, equivalent to the pressure on the Earth's surface with a slightly elevated $\mathrm{CO}_{2}$ level. The range of inside temperature is actively maintained between $18^{\circ} \mathrm{C}$ and $26^{\circ} \mathrm{C}$. The 8 wings of solar arrays are capable of generating up to $84 \mathrm{~kW}$ of power, most of which is used by the vessel's systems and payloads or otherwise used to charge batteries. The available electrical energy, on the United States Orbital Segment side, is a stabilized $124 \mathrm{~V}$ and power outlets are widely available. Data connections are available via buses (MIL 1553) or LAN cables (Ethernet 802.3). Telemetry downlink and telecommand and data uplink are available via the Transmitter Data Service Request constellation and in the $\mathrm{Ku}$ and $\mathrm{S}$ frequency band. The ISS can also stream live video channels for scientific purposes.

The microgravity conditions aboard the ISS are the following:

\section{- $\quad$ Residual acceleration at the center of mass: $3.9 \times 10^{-7} \mathrm{~g}$ \\ - The level of microgravity decreases as one moves away from the center of mass of the structure.}

All technical aspects mentioned above, such as the power consumption, telecommunication, medical surveillance of the subjects, weight etc., have to be taken into account in case a short radius centrifuge is planned to be installed aboard the ISS or any other space vessel. Though the power supply, the medical surveillance, the various environmental conditions and the vibration generated by such a device are issues that should not be neglected, the matter of utmost importance is obviously the space. Since the room onboard a space vessel even as vast as the ISS is scarce, the fitting of a short radius centrifuge in a diameter of any module and the placing of such a device requires a thorough study of volume envelope $v s$ subject posture. Such a device would be installed aboard the ISS in order to counteract the negative effects of microgravity during long space missions [13].

\section{LIFE SCIENCES ASPECTS}

\section{Effects of AG and the Gravity Gradient Generated by a Short Radius Centrifuge on the Human Physiology}

The sojourn in the microgravity environment results not only in immediately detectable changes such as the neurocognitive impairments but also in mid-term and longterm deconditioning of physiological structures such as the cardiovascular system and the musculoskeletal system. These changes are confirmed by a number of studies, which assessed the astronaut's cardiovascular system prior, during and post long-term sojourn in microgravity [14]. Thus, strict physical activity protocols and a number of various countermeasures are applied in order to maintain the integrity of, e.g., the cardiovascular system and the musculoskeletal system and consequently the general physical fitness. The current ESA and DLR strategy involves the application of AG in order to counteract cardiovascular, metabolic, cellular and musculoskeletal deconditioning. The application of AG generated by short radius centrifuges came even more into focus due to current plans for interplanetary missions to the Moon and Mars [15].

Thus, the application of AG generated by short radius centrifuges is under intense assessment in Europe and elsewhere in the world ([e.g. [4]). The rotation of at least one subject in supine or sitting position can generate a centrifugal force which simulates the gravity-induced physiological effects while standing at a certain $g$-load. At the same time a short radius centrifuge generates a severe gravitational gradient in the head-to-toe axis, which considerably effects cardio-vegetative regulation and haemodynamics in humans. Therefore, future projects should aim to expand the scientific knowledge of effects of AG on the cardiovascular system, the ANS, the musculoskeletal system, the cognitive perception and the cellular functions [16]. These assessments ought to have an essential impact for future in-spaceflight and post-spaceflight countermeasures, since the application of SAHC devices have been proposed by a number of space agencies and scientists (e.g., [17]) in order to expose astronauts to AG in the course of sojourn in microgravity.

Hence, the SAHC is proposed as a potential countermeasure method against degradation of various physiological structures and cellular systems. Though budding devices, the short radius centrifuges are not ready for practical countermeasure applications yet, since there is a considerable lack of knowledge as far as physiological and cellular effects of AG generated by SAHC devices is concerned.

\section{Heart Rate Variability - A Central Parameter for Life Science Experiments}

The relationship between microgravity related cardiovascular decompensation and HRV have been assessed during few previous studies [18, 19], since the HRV represents the vegetative regulation of the cardiovascular system [20-22]. The HRV is an important and easy to measure indicator of cardiovascular function and has been assessed during gravity transitions [20]. Seps et al. reported (2002) Sasaki et al. reported (1999) a strong relationship between AG ( $2 g$ at the level of feet), applied by a short 
radius centrifuge and a significantly reduced cardiovascular degradation during sojourn in microgravity.

HRV is an imminent expression of sympathetic and parasympathetic influences of the function of the heart [23]. Arrhythmias, which are essential for standard diagnostics in the field of medicine and sportsmedicine [24] vary between non-hazardous respiratory sinus arrhythmias (RSA) and chronicle atrial fibrillation. This important parameter has considerable potential in assessing the role of ANS functions in healthy individuals and patients [25]. The HRV is defined as variation and fluctuation of heart rate during a certain period of time [26]. RSA is one of the principle components of HRV. Migeotte et al. (2009) demonstrated that the effects of hypergravity or AG, respectively, on RSA can mimic the effects observed after spaceflight cardiovascular deconditioning. The reason for this effect is the significant gravity gradient along the head-to-toe axis, which occurs when AG is generated by a short radius centrifuge. Data from Migeotte (2009) suggest that RSA and therefore HRV change under hypergravity conditions due to venous pooling in the peripheral limbs, the subsequent reduced filling of the right atrium and the autonomic adaptation. In their conclusion Migeotte et al. (2009) call for additional assessments and further studies addressing both changes in the overall $g$-load and the gravity gradient to find a proper $g$ load for this countermeasure method and in order to evaluate the dose-response of the gradient effect. Furthermore, data from [6] suggest changing the angle of the SAHC or experiments during hyper- $g$ phases of a parabolic flight in order to modify/eliminate the gradient in the gravitational field. Since gravity in general and hyper- and microgravity in particular have an influence on various aspects of the cardiovascular system and the ANS, upright positioning (under $1 g$ condition) usually causes sub diaphragmatic gravitation blood pooling primarily within the venous system [27]. As a result, the venous return to the right atrium and the cardiac output subsequently decrease in all upright objects [28] and in supine posture, respectively, during centrifugation. Tilt table tests (TTT) studies in subjects with postural fainting resulted in excessive splanchnic pooling. Thoracic hypovolaemia and increased peripheral resistance appear immediately before syncope [29]. The HRV data which were recorded during the same study support the hypothesis of higher sympathetic activation in fainters soon after head up tilt (HUT) with sympathetic withdrawal and parasympathetic activation just before fainting. Intravascular pooling mainly within the splanchnicus, the pelvis and the legs is a commonly regarded phenomenon of space related experiments with devices such as SAHC, LBNP and TTT [28]. Stasis of circulation, especially insufficient venous reflux, the decreased filling of the right atrium and a reduction in arterial resistance are the main risk factors for orthostatic related loss of consciousness [31, 32] and spontaneous venous thrombosis with fatal consequences [33]. Plans to implement a HRV measurement as a solid control tool for user applied short radius centrifugation at the SAHC at DLR are currently under way.

Short radius centrifuges are a novel concept of preventing the decaying effects of long term weightlessness on humans. Therefore, the feasibility of a SAHC on a space vessel is an object of interest for space medicine as well as space engineering. Regarding the benefits of AG applied by a SAHC device the risks for astronauts or test subjects here on Earth should not be neglected.

\section{Application of Short Radius Centrifuges in Basic Biomedical Research}

The supine table of the SAHC (Fig. 4) serves plenty of room for biotechnological or cell biological experiments. The effects of AG on the response of immune cells have recently been under investigation. A Photomultiplier (PMT) 2D-clinostat (Fig. 5) was implemented on the supine position of the SAHC. This experimental setup (Fig. 4) enabled online kinetic luminescence assays during hypergravity. First results on the activity of immune cells during centrifugation have been published [34].

The clinostat, usually designed as a microgravity simulator, was used as a measurement device on the SAHC as well as during parabolic flights. With the assistance of a special computer program and a relay box, the clinostat and centrifuge can be employed consecutively in order to perform a ground-based parabolic flight like $g$-profile.

Further insight on cell morphology and cellular responses can be gained with the use of the SAHC, due to the advantage of online monitoring instead of commonly used end point measurements. Multiwell plate readers can be installed on the supine position of the centrifuge in order to explore different parameter according to specialized absorbance, fluorometric and luminescent assays. Furthermore, online observation of the cell morphology with confocal laser scanning microscope technique is currently in preparation. Multiwell plate reader as well as microscope is to be installed on a rocker on the SAHC to ensure the $g$-force acting perpendicular on the attached cells $[34,35]$.

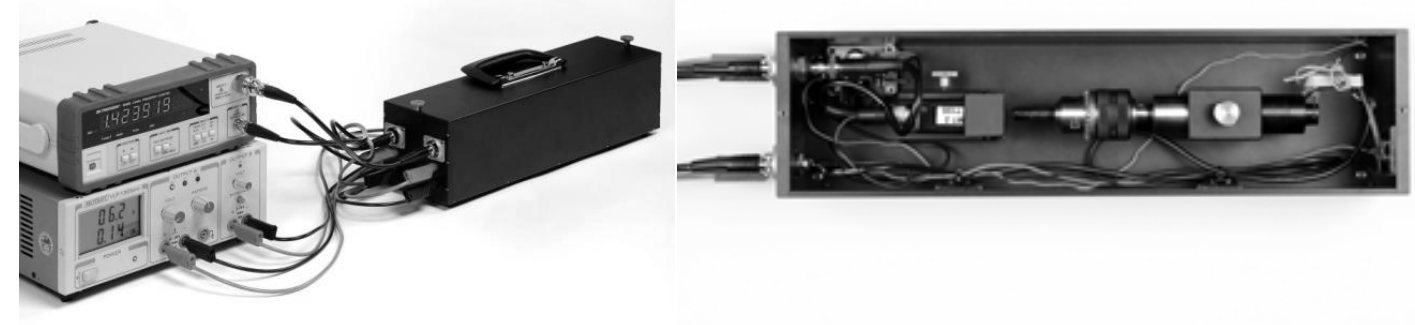

Fig. (4). Photomultiplier clinostat (black box), frequency counter and power supply (left) and details (PMT, cuvette, motor) (right). 


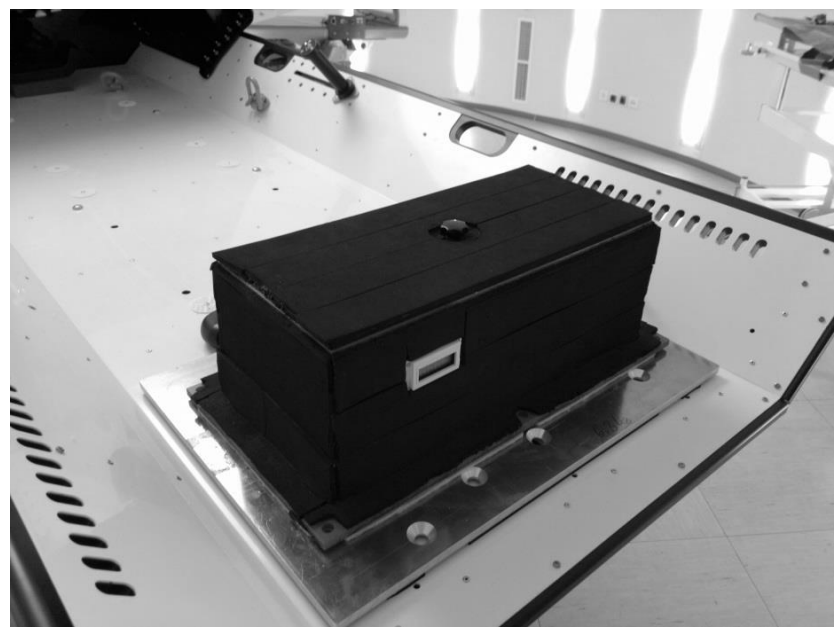

Fig. (5). The heating box installed on a supine position of the SAHC. The PMT clinostat is located within the heating box.

Overall, short radius centrifuges such as the SAHC at the DLR, Cologne, provide a platform for carrying out a variety of experiments addressing human-physiological issues in order to yield clues and insights regarding possible countermeasures against effects of weightlessness using AG during prolonged space flight. Moreover, such centrifuges can easily be adapted to be used in basic biomedical research in carrying devices containing, e.g., cell biological experiments.

\section{CONFLICT OF INTEREST}

The authors confirm that this article content has no conflict of interest.

\section{ACKNOWLEDGEMENTS}

This work was supported by the ESA, the DLR Institute of Aerospace Medicine and the German Sport University Cologne (DSHS) Institute of Cardiology and Sports medicine. The authors also thank the subjects who participated in the experiments and studies, the SAHC team for the operational guidance as well as the Studio Michael Najjar for the use of the graphical material in this manuscript.

\section{REFERENCES}

[1] G. Clèment, A. Pavy-Le Traon, "Centrifugation as a countermeasure during actual and simulated spaceflight: A review", Eur J Appl Physiol, Vol.92, pp. 235-248, 2004.

[2] I. Retat, “Micro-g neutral platform for space travel missions," U.S Patent US5098040 A, 1992.

[3] G. Mullenburg, J. Vernikos, "Human powered centrifuge," U.S Patent US5616104 A, 1997.

[4] G. Clèment, K. Slenzka, "Fundamentals of Space Medicine", Microcosm Press, El Segundo and Springer, Dordrecht. 2006.

[5] L.R. Young, H. Hecht, L.E. Lyne, K.H. Sienko, C.C. Cheung, J. Kavelaars, "Artificial gravity: head movements during short-radius centrifugation", Acta Astronaut., Vol. 49(3-10), pp. 215-26, 2001.

[6] P.F. Migeotte, N. Pattyn, R. Vanspauwen, X. Neyt, M. Acheroy, P. Van de Heyning, F.L. Wuyts, "Respiratory sinus arrhythmia on the ESA-short-arm human centrifuge", IEEE Eng Med Biol Mag., Vol. 28(6), pp. 86-91, 2009.

[7] T. Sasaki, K.I. Iwasaki, K. Hirayanagi, N. Yamaguchi, A. Miyamoto, K. Yajima, "Effects of daily 2-Gz load on human cardiovascular function during weightlessness simulation using 4day head-down bed rest", Vol. 36(3), pp. 113-23, 1999.

[8] R.S. Johnston, L.F. Dietlein, "Biomedical Results from Skylab", NASA SP-377, Washington D.C.: NASA, 1977.

[9] A.J. Benson, F.E. Guedry, D.E. Parker, MF. Reschke, "Microgravity vestibular investigations: perception of selforientation and self-motion", J Vestib Res, Vol. 7, pp. 453-457, 1997

[10] S.T. Moore, G. Clement, T. Raphan, B. Cohen, "The human response to artificial gravity in a weightlessness environment: Results from the Neurolab centrifugation experiments", In: ElGenk MS. Space Technology and Applications International Forum 2000. American Institute of Physics, College Park, pp 206-211, 2000.

[11] S. Moore, G. Clèment, T. Raphan, B. Cohen, "Ocular counterrolling induced by centrifugation during orbital spaceflight", Exp Brain Res, Vol. 137, pp. 323-335, 2001.

[12] A.I. Grigoriev, V.M. Baranov, V.V. Bogomolov, O.G. Gazenko, V.A. Knyazhev, Y.V. Natochin, S.O. Nikolaev, V.V. Polyakov, G.I. Samarin, "Orbital Station Mir: Space Biology and Medicine", Vol. 1 (in Russian). Moscow: IMBP, 2001.

[13] A.R. Kotovskaia, I.F. Will-Williams, "The short-arm centrifuge: history and possible uses in cosmonautics and health care services", Aviakosm Ekolog Med., Vol. 38(5), pp. 3-11, 2004.

[14] A.R. Hargens, R. Bhattacharaya, S.M. Schneider, "Space physiology VI: exercise, artificial gravity, and countermeasure development for prolonged space flight”, Eur J Appl Physiol., 2012 Oct 19. [Epub ahead of print], 2012.

[15] S. Lazzer, L. Plaino, G. Antonutto, "The energetics of cycling on Earth, Moon and Mars”, Eur J Appl Physiol., Vol. 111(3), pp. 35766, 2011.

[16] A. Bukley, D. Lawrence, G. Clément, "Generating artificial gravity onboard the Space Shuttle", Acta Astronautica, Vol. 60, pp. 472478, 2007.

[17] T. Sasaki, K.I. Iwasaki, K. Hirayanagi, N. Yamaguchi, A. Miyamoto, K. Yajima, "Effects of daily 2-Gz load on human cardiovascular function during weightlessness simulation using 4day head-down bed rest", Vol. 36(3), pp. 113-23, 1999.

[18] F. Iellamo, M. Di Rienzo, D. Lucini, J.M. Legramante, P. Pizzinelli, P. Castiglioni, F. Pigozzi, M. Pagani, G. Parati, "Muscle metaboreflex contribution to cardiovascular regulation during dynamic exercise in microgravity: insights from mission STS-107 of the space shuttle Columbia”, J Physiol., Vol. 1;572(Pt 3), pp. 829-38, 2006.

[19] J. Tank, R.M. Baevsky, I.I. Funtova, A. Diedrich, I.N. Slepchenkova, J. Jordan, "Orthostatic heart rate responses after prolonged space flights", Vol. 21(2):121-4. Epub 2010 Dec 25, 2011.

[20] B. Seps, F. Beckers, A.E. Aubert. "Heart rate variability during gravity transitions", Comput Cardiol. Vol. 29, pp. 433-6, 2002.

[21] F. Beckers, B. Seps, D. Ramaekers, B. Verheyden, A.E. Aubert. "Parasympathetic heart rate modulation during parabolic flights", Eur J Appl Physiol., 90(1-2):83-91. Epub 2003 Jun 13, 2002.

[22] K.I. Iwasaki, T. Sasaki, K. Hirayanagi, K. Yajima, "Usefulness of daily $+2 \mathrm{Gz}$ load as a countermeasure against physiological problems during weightlessness", Acta Astronaut., Vol. 49(3-10), pp. 227-35, 2001.

[23] Task Force of the European Society of Cardiology and the North American Society of Pacing and Electrophysiology, "Heart Rate Variability: standards of measurement, physiological interpretation and clinical use" Circulation, Vol. (93), pp. 1043-1065, 1996 / Eur Heart J, Vol. 17(3), pp. 354-81, 1996.

[24] J. Latsch, H. Predel, "Cardiac arrhythmia in sports Herz", Review. German Jun; Vol. 29(4), pp. 420-5, 2004.

[25] X. Yan, F. Zhang, "Analysis of heart rate variability and its application”, Space Med Med Eng (Beijing). 1997 Vol. 10(5), pp. 318-22, 1997.

[26] K. Hottenrott, "Herzfrequenzvariabilität im Sport. PräventionRehabilitation-Training".Schriften der Deutschen Vereinigung für Sportwissenschaft, 2001.

[27] L.B., "Rowell Human Cardiovascular Control", New York: Oxford University Press, 1993.

[28] D. O'Leary, D. Kimmerly, A. Cechetto, J. Shoemaker, "Differential effect of head-up tilt on cardiovagal and sympathetic baroreflex sensitivity in humans", Exp Physiol, Vol. 88, pp. 769-774, 2003. 
[29] I. Taneja, M.S. Medow, J.L. Glover, N. Raghunath, M. Stewart. "Increased vasoconstriction predisposes to hyperpnea and postural faint", Am J Physiol Heart Circ Physiol., Vol. 295(1), pp. H372$\mathrm{H} 381,2008$.

[30] R.L. Summers, S. Platts, J.G. Myers, T.G. Coleman, "Theoretical analysis of the mechanisms of a gender differentiation in the propensity for orthostatic intolerance after spaceflight," Theor Biol Med Model., Vol. 18, pp. 7:8, 2010.

[31] G. Fucà, M. Dinelli, P. Suzzani, S. Scarfò, F. Tassinari, P. Alboni, "The venous system is the main determinant of hypotension in patients with vasovagal syncope," Europace., Vol. 8(10), pp. 83945. Epub 2006 Aug 17, 2006

[32] B. Verheyden, F. Beckers, K. Couckuyt, J. Liu, A.E. Aubert, "Respiratory modulation of cardiovascular rhythms before and after short-duration human spaceflight," Acta Physiol (Oxf)., Vol. 191(4), pp. 297-308. Epub 2007 Sep 3, 2007.

[33] M. Masoud, G. Sarig, B. Brenner, G. Jacob, "Orthostatic hypercoagulability: a novel physiological mechanism to activate the coagulation system", Hypertension., Vol. 51(6), pp. 1545-51. Epub 2008 Apr 14, 2008.

[34] A. Horn, O. Ullrich, K. Huber, R. Hemmersbach, !PMT (Photomultiplier) clinostat“, Microgravity Sci Technol, Vol. 23(1), pp. $67-71,2010$.

[35] A. Horn "Vorbereitungen für das Biolab Experiment TRIPLE LUX A Hardwareentwicklung, Kalibrierung und biologische Bodenkontrollen“, thesis, Fakultät für Maschinenbau, University of Magdeburg, Germany, 2011. 